\title{
ARtigos
}

\section{Mortalidade por homicídios em Londrina, município do norte do Paraná, no período entre 2006 e 2008}

\author{
Homicide mortality in Londrina, a city of southern \\ Brazil, 2006-2008
}

Olavo Franco Ferreira Filho(1), Alcindo Cerci Neto(2)

Ferreira Filho OF, Cerci Neto A. Mortalidade por homicídios em Londrina, município do norte do Paraná, no período entre 2006 e 2008. Saúde, Ética e Justiça. 2010;15(2):63-8.

RESUMO: Os homicídios constituem uma importante causa de morte prematura e a mortalidade por este tipo de violência vem aumentando em todo o mundo nos últimos anos. Esta pesquisa teve por objetivo traçar o perfil epidemiológico dos homicídios em Londrina, norte do Paraná. Trata-se de um estudo descritivo onde foram avaliadas retrospectivamente as necropsias do Instituto Médico Legal (IML) da cidade de Londrina-PR no período de julho de 2006 a junho de 2008. Neste período o IML realizou 675 necropsias de vítimas residentes naquela cidade, sendo $261(38,7 \%)$ decorrentes de homicídio, equivalendo a uma taxa de 53 mortes para cada 100 mil habitantes. Não foi possível encontrar informações completas em 29 casos (4,3\%). Dos 232 casos analisados, havia $220(94,8 \%)$ homens e 12 (5,2\%) mulheres. A relação de homens e mulheres foi de 18:1. A maioria das vítimas tinha cor branca $(65,1 \%)$ e era solteira $(78,9 \%)$. As idades variaram de 7 até 79 anos (mediana de 24 anos). De acordo com tipo de homicídio encontramos: $87,5 \%$ de ferimentos por arma de fogo e $12,5 \%$ de outros ferimentos. Em $200(86,2 \%)$ vítimas foi realizada pesquisa toxicológica e alcoólica. Nas 59 (25,4\%) vítimas que apresentavam dosagem alcoólica positiva, a média de álcool encontrada foi de 15,2 +7,0 dg/l com mediana de 14,6 dg/l. A taxa de mortalidade por homicídios e o uso de armas de fogo na cidade de Londrina é alarmante, retirando da sociedade pessoas jovens e gerando um impacto negativo na esperança de vida.

DESCRITORES: Homicídio/estatística \& dados numéricos; Epidemiologia; Coeficiente de mortalidade; Armas de fogo.

(1) Docente Associado do Departamento de Clínica Médica da Universidade Estadual de Londrina. Coordenador do Departamento de Clínica Médica.

(2) Docente Adjunto A do Departamento de Clínica Médica da Universidade Estadual de Londrina. Coordenador da Disciplina de Medicina Forense.

Endereço para correspondência: Alcindo Cerci Neto. Rua Senador Souza Naves, 2677 - Londrina, Paraná. CEP: 86015430. E-mail: alcindoneto@uel.br. 


\section{INTRODUÇÃO}

U m estudo sobre violência realizado pela Organização das Nações Unidas (ONU), discutido em evento na cidade de Duban, África do Sul, em 2008, mostra que os casos de homicídio em todo o mundo cresceram $30 \%$ entre 1980 e 2000 , passando de 2.300 para mais de 3.000 por 100 mil pessoas. É na América Latina e Caribe e na África Subsaariana que estão as mais altas taxas deste tipo de violência, enquanto a União Européia e os Estados Árabes têm as mais baixas. A comparação entre os países aponta que Colômbia, África do Sul, Jamaica, Guatemala e Venezuela têm os índices de homicídio mais altos, enquanto Japão, Arábia Saudita, Catar, Espanha, Chipre e Noruega têm os mais baixos. Mas, segundo o estudo, Washington DC, nos Estados Unidos, concentra as taxas mais altas de assassinatos do mundo ${ }^{1}$.

Em escala mundial, no ano 2000 ocorreram 520.000 mortes por homicídio correspondendo a $31 \%$ dos óbitos violentos e a um coeficiente de 8,8 por 100 mil habitantes. No Brasil, no mesmo ano, a taxa de mortalidade por homicídio foi de 28 mortes para cada 100 mil habitantes ${ }^{2}$, inferior somente a da Colômbia, onde a taxa foi de 60 para cada 100 mil habitantes ${ }^{3}$. Assim, observamos que este tipo de morte violenta constitui um grave problema pela sua elevada freqüência.

Os assassinatos são mais freqüentes em adolescentes e jovens do sexo masculino, por estarem mais expostos a violência, o que gera um impacto negativo na esperança de vida dos brasileiros $^{4}$, além de graves repercussões sobre o sistema de saúde, a previdência social e o judiciário.

Não encontramos estudo sobre este tema referente ao estado do Paraná. Assim, o presente levantamento tem como objetivo traçar o perfil epidemiológico dos homicídios na cidade de Londrina, bem como determinar a taxa de mortalidade por este tipo de violência nesta cidade.

\section{METODOLOGIA}

Londrina, localizada no norte do Paraná, é a terceira mais importante cidade do sul do Brasil, com característica econômica centrada principalmente na prestação de serviços, no agronegócio e no comércio, tendo uma população estimada para 2008 de 500.000 habitantes $^{5}$. Nesta cidade são realizadas, pelos peritos do Instituto Médico Legal (IML), todas as necropsias de vítimas de morte violenta da macrorregião. Amostras de sangue e tecido são coletadas e encaminhadas ao laboratório de toxicologia do IML de Curitiba para as análises pertinentes, de acordo com a suspeita do caso.

Foram analisados retrospectivamente todos os laudos de necropsia do período de julho de 2006 a junho de 2008, de vítimas residentes na cidade de Londrina, cuja morte foi decorrente de homicídio. Foram coletados dados demográficos e relativos ao tipo de assassinato, local e horário do ocorrido, local de morte, segmento corporal agredido e dosagem alcoólica da vítima. Com relação ao horário da ocorrência, foi considerado: madrugada (das 24:01 às 7:00 horas); período matutino (das 7:01 às 13:00 horas); período vespertino (das 13:01 às 19:00 horas) e período noturno (das 19:01 às 24:00 horas). Não foram incluídos os laudos incompletos que aguardavam exame toxicológico.

Utilizou-se a estatística descritiva para demonstrar os resultados.

\section{RESULTADOS}

No período de estudo, o IML realizou 675 necropsias de vítimas residentes em Londrina, sendo 261 (38,7\%) decorrentes de homicídio, equivalendo a uma taxa de 53 mortes para cada 100 mil habitantes. Não foi possível encontrar informações completas em 29 casos (4,3\%). Dos 232 casos analisados, havia 220 (94,8\%) homens e $12(5,2 \%)$ mulheres. A relação de homens e mulheres foi de 18:1. Com relação à cor da pele, encontramos 151 (65,1\%) brancos e 81 (34,9\%) não brancos. As idades das vítimas variaram de 7 até 79 anos (mediana de 24 anos). Com relação ao estado civil, 183 (78,9\%) eram solteiros. A Figura 1 mostra a distribuição das vítimas estratificadas pela idade.

De acordo com o tipo de homicídio, encontramos: $203(87,5 \%)$ ferimentos por arma de fogo, $18(7,8 \%)$ agressões físicas com objetos contundentes diversos e $11(4,7 \%)$ ferimentos por arma branca. O número de perfurações encontradas nos segmentos corporais variou de 1 a 19 (mediana = 3), sendo que, quando o crânio era o alvo das agressões, havia menos de 3 orifícios de entrada. A tabela 1 mostra outras características das vítimas de homicídio.

Em 200 (86,2\%) vítimas foi realizada pesquisa toxicológica e alcoólica. Nas 59 (25,4\%) vítimas que apresentavam dosagem alcoólica positiva, a média de álcool encontrada foi de 15,2 \pm 7,0 dg/l com mediana de 14,6 dg/l. Em uma vítima a pesquisa para cocaína teve resultado positivo. 
Ferreira Filho OF, Cerci Neto A. Mortalidade por homicídios em Londrina, município do norte do Paraná.

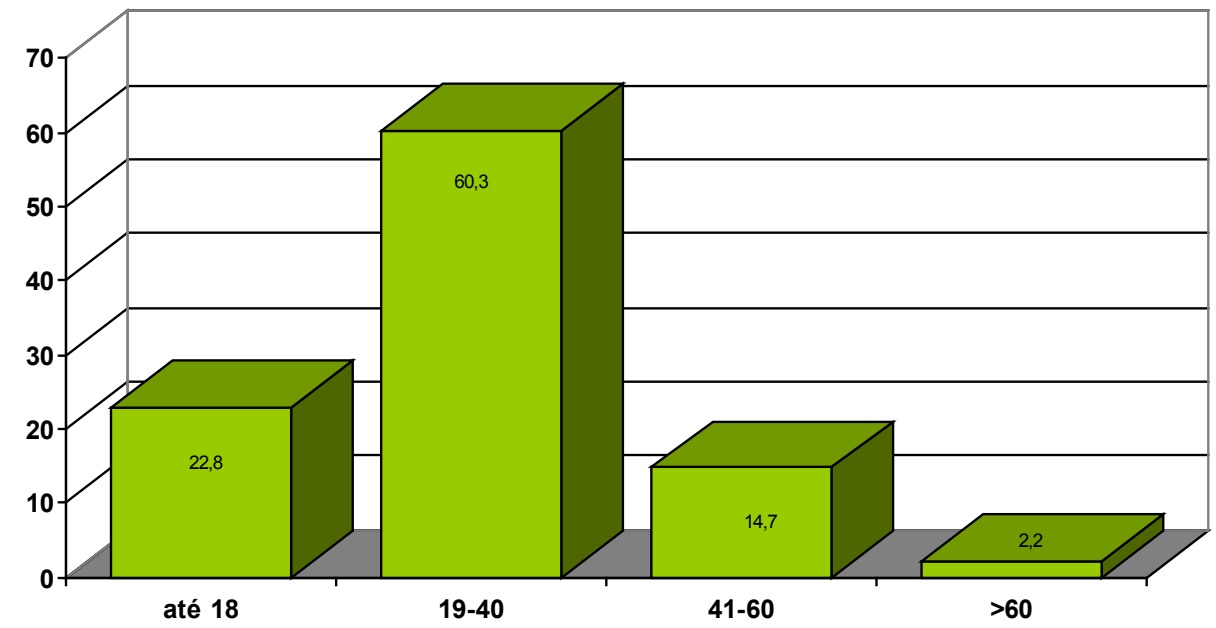

Figura 1: Distribuição (\%) das vítimas de homicídio da cidade de Londrina, no período de julho de 2006 a junho de 2008, de acordo com a faixa etária (em anos)

Tabela 1: Características das vítimas de homicídio da cidade de Londrina no período de julho de 2006 a junho de 2008

\begin{tabular}{|c|c|c|}
\hline Parâmetro & $\mathbf{N}$ & $\%$ \\
\hline 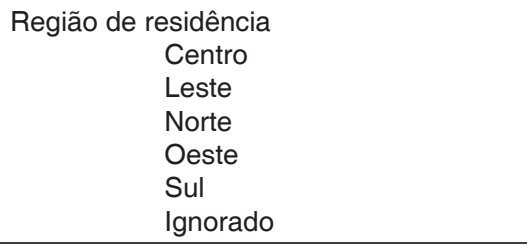 & $\begin{array}{l}30 \\
40 \\
69 \\
36 \\
51 \\
6\end{array}$ & $\begin{array}{l}12,9 \\
17,2 \\
29,7 \\
15,5 \\
22,0 \\
2,6\end{array}$ \\
\hline $\begin{array}{l}\text { Região da ocorrência } \\
\text { Centro } \\
\text { Leste } \\
\text { Norte } \\
\text { Oeste } \\
\text { Sul } \\
\text { Fora da cidade } \\
\text { Ignorado } \\
\end{array}$ & $\begin{array}{l}23 \\
28 \\
54 \\
32 \\
33 \\
17 \\
45\end{array}$ & $\begin{array}{c}9,9 \\
12,1 \\
23,3 \\
13,8 \\
14,2 \\
7,3 \\
19,4 \\
\end{array}$ \\
\hline $\begin{array}{l}\text { Horário da ocorrência } \\
\qquad \begin{array}{l}\text { Na madrugada } \\
\text { Período matutino } \\
\text { Período vespertino } \\
\text { Período noturno } \\
\text { Ignorado }\end{array}\end{array}$ & $\begin{array}{c}41 \\
26 \\
50 \\
112 \\
3\end{array}$ & $\begin{array}{c}17,7 \\
11,2 \\
21,6 \\
48,3 \\
0,2\end{array}$ \\
\hline $\begin{array}{l}\text { Local da ocorrência } \\
\qquad \begin{array}{l}\text { Residência ou próprio bairro } \\
\text { Outro bairro } \\
\text { Ignorado }\end{array}\end{array}$ & $\begin{array}{l}131 \\
53 \\
48\end{array}$ & $\begin{array}{l}56,5 \\
22,8 \\
20,7\end{array}$ \\
\hline $\begin{array}{l}\text { Local do óbito } \\
\qquad \begin{array}{l}\text { Hospital } \\
\text { Local da ocorrência }\end{array}\end{array}$ & $\begin{array}{c}50 \\
182 \\
\end{array}$ & $\begin{array}{l}21,6 \\
78,4 \\
\end{array}$ \\
\hline $\begin{array}{l}\text { Segmento corporal agredido* } \\
\text { Crânio } \\
\text { Pescoço } \\
\text { Tórax } \\
\text { Abdome } \\
\text { Membros } \\
\end{array}$ & $\begin{array}{c}90 \\
15 \\
116 \\
46 \\
1\end{array}$ & $\begin{array}{c}38,8 \\
6,5 \\
50,0 \\
19,8 \\
0,4\end{array}$ \\
\hline $\begin{array}{l}\text { Dosagem alcoólica } \\
\text { Positiva } \\
\text { Negativa } \\
\text { Ignorado }\end{array}$ & $\begin{array}{c}59 \\
141 \\
32\end{array}$ & $\begin{array}{l}25,4 \\
60,8 \\
13,8\end{array}$ \\
\hline
\end{tabular}

* 35 (15,1\%) vítimas tiveram lesão em mais de um segmento corporal 


\section{DISCUSSÃO}

O homicídio atualmente é uma das principais causas de morte precoce em todo o mundo, acometendo mais frequentemente homens jovens, gerando grande impacto nos indicadores de nível de saúde como os Anos Potenciais de Vida Perdidos.

Encontramos, em nossa pesquisa, uma taxa de mortalidade por homicídio de 53 casos para cada 100 mil habitantes, taxa bastante alta, considerando que a média nacional, segundo Soares Filho e colaboradores, é de 28 óbitos para cada 100 mil habitantes ${ }^{2}$. Porém, este autor estudou o período de 1980 a 2003 e usou a como referência a população brasileira do ano 2000, o que faz com que seus dados possam ser considerados desatualizados, tendo em vista que outros estudos mostram tendência ao aumento das taxas de homicídio nos últimos anos ${ }^{6}$.

Com relação à idade, $60,3 \%$ dos óbitos ocorreram na faixa etária dos 19 aos 40 anos e quase $100 \%$ das vítimas eram homens e $78,9 \%$ eram solteiros. Valores semelhantes foram encontrados por Hennington et al. ${ }^{7}$ quando estudaram a mortalidade por homicídios na cidade de São Leopoldo, Rio Grande do Sul, no período de 1996 a 2005 e por Barata et al. ${ }^{8}$ estudando a violência na cidade de São Paulo.

Segundo Souza ${ }^{9}$, para os adolescentes e adultos jovens vivendo em condições de exclusão social, os comportamentos violentos aparecem como reafirmadores de identidade, levando este grupo etário a predominar entre os agentes e alvos da violência. A frustração das expectativas associada ao desemprego crônico e à falta de participação na vida social acabam por produzir sentimentos de marginalização e diminuição da auto-estima, concorrendo para maior exposição e vitimização.

Nosso estudo mostrou que ocorreu maior freqüência de homicídios entre as vítimas de cor branca $(65,1 \%)$. Outros trabalhos mostram que o risco de homicídio é maior nos indivíduos negros quando comparado aos brancos, associando-se este fato a pior condição social ${ }^{6,7,8}$.

0 instrumento mais frequentemente utilizado para a agressão foi a arma de fogo, o que correspondeu a $87,5 \%$ dos casos. Este tipo de arma é relatado pela maioria das pesquisas sobre o tema ${ }^{5,6}$. Peres e Santos ${ }^{6}$, estudando 13 capitais brasileiras, mostraram que, em 1991, 50\% dos homicídios foram produzidos por armas de fogo e, em 2000 , esta taxa subiu para $70 \%$. Mostraram, também, que o coeficiente de mortalidade por homicídio cresceu $27,5 \%$ sendo que o incremento nos homicídios cometidos por arma de fogo foi de $72,5 \%$. Em nossa pesquisa não observamos, nos boletins policiais de ocorrência, qualquer referência de que a arma apreendida no momento do crime fosse legalizada, mostrando a alta clandestinidade deste tipo de armamento.

Com relação ao local de moradia e ao local da ocorrência do homicídio, encontramos que os moradores de região norte e sul foram os mais vitimados. Isso é explicado porque, em Londrina, nestas regiões ocorre uma maior concentração populacional, bem como um maior número de indivíduos de baixa condição social. Macedo et al. ${ }^{10}$ encontraram taxas de mortalidade por homicídios mais elevadas nas áreas mais pobres de Salvador. Barata et al. $^{8}$, estudando a associação entre as desigualdades sociais e os homicídios, mostraram que no estrato $A$ da sociedade os homicídios estavam associados a assaltos; no estrato $B, C$ e $D$, relacionavam-se ao tráfico de drogas e a outras atividades ilegais e, no estrato E, a maioria estava associada a brigas e desavenças entre indivíduos. Isso pode explicar, também, o fato observado em nosso estudo de que a maioria dos assassinatos ocorre no período noturno, horário em que mais frequentemente ocorrem assaltos e brigas entre os indivíduos.

Não pudemos precisar o local da ocorrência, se era um local público ou a própria residência. Observamos que $56,5 \%$ deles ocorreram na residência da vítima ou próximo a ela. Em São Paulo, os trabalhos mostram maior ocorrência em locais públicos (bares e ruas) e menos de 10\% na própria residência ${ }^{8}$. Em Nova York, cerca de $30 \%$ dos homicídios ocorrem nos domicílios ou locais de trabalho, e $45 \%$ em locais públicos ${ }^{11}$.

Quase $80 \%$ das vítimas morreram no local sem que houvesse tempo para que recebessem cuidados médicos, mostrando a gravidade das ocorrências. Observamos que os segmentos corporais mais agredidos foram o crânio e o tórax, regiões de órgãos nobres e fundamentais para a vida. Lesões corporais semelhantes são descritas por outros autores ${ }^{8}$.

Em 13,8\% dos casos a pesquisa toxicológica não foi realizada. Estes são aqueles indivíduos encaminhados a hospital e não é protocolo nos hospitais de Londrina coletar exames toxicológicos em vítimas de violência. Em 25,4\% de nossa amostragem a dosagem alcoólica foi positiva, sendo a média de 15,2 dg/l, bem acima do limite 
Ferreira Filho OF, Cerci Neto A. Mortalidade por homicídios em Londrina, município do norte do Paraná.

de nossa legislação, que considera tolerável até $6 \mathrm{dg} / \mathrm{I}^{12}$. Apesar de outros trabalhos ${ }^{8}$ mostrarem a associação de homicídios com tráfico de drogas, em nosso trabalho apenas em uma vítima a pesquisa para cocaína foi positiva.

Assim, observamos que e em Londrina a taxa de mortalidade por homicídios é alta, tirando da sociedade indivíduos jovens e em plena produtividade intelectual e econômica. Souza ${ }^{13}$ assinala três fatos relacionados a este tipo de violência: 1) a consolidação da organização do crime, com uma economia e um poder paralelo, constituindo uma rede articulada e difícil de ser controlada; 2) consolidação dos grupos de extermínio; 3) aumento da pobreza e da miséria urbanas, principalmente na população em idade produtiva, vivendo e trabalhando nas ruas, e de uma população infantil e juvenil compelida ao trabalho e à busca de sobrevivência acrescida da falência das instituições de atenção a crianças e adolescentes.

Para a superação da violência da atualidade, incluindo o elevado número de homicídios, deve-se ampliar o enfoque sobre o fenômeno, considerandose as condições de vida e de trabalho e, além disso, avançar no entendimento do modo de vida do jovem brasileiro, fragilizado pelos reveses sociais e econômicos e pela degradação dos padrões familiares.

Ferreira Filho OF, Cerci Neto A. Homicide mortality in londrina, a city of southern Brazil, 2006-2008. Saúde, Ética e Justiça. 2010;15(2):63-8.

ABSTRACT: Homicide is an important cause of premature death and the mortality rate is increasing in the world. The aim of this study is to outline the epidemiological profile of homicides in the city of Londrina, north of the state of Paraná, southern Brasil. A retrospective study of homicide cases were undertaken at the Londrina Coroner Department fromJuly 2006 to June 2008. A total of 232 cases were found, with $220(94.8 \%)$ males and $12(5.2 \%)$ females. The male and female ratio was 18:1. Age range from 7-79 years; median 24 years. There were $151(65.1 \%)$ white and $183(78.9 \%)$ were single. Homicide mortality rate were 53 deaths per 100.000 habitants. The homicides were caused byfirearms $(87.5 \%)$ and non-firearms (12.5\%). In 200 victims was possible to held a toxicological exams; in 59 (25.4\%) alcoholic dosage was positive; mean $15.2 \pm 7.0 \mathrm{dg} /$; median of $14.6 \mathrm{dg} / \mathrm{l}$. The mortality rate in city of Londrina displays alarming profile-higher rates, greater percentage of firearms use and higher rates among male youth, created a negative impact of life's expectations.

KEY WORDS: Homicide/statistics \& numerical data; Epidemiology, Mortality rate; Firearms.

\section{REFERÊNCIAS}

1- Organização das Nações Unidas (ONU). International youth crime prevention \& cities summit (UN-HABITAT), trends \& types of crime and violence. Durban, South Africa, 2008 [cited 2008 Nov]. Available from: http://www.unhabitat.org/ downloads/docs.

2- Soares Filho AM, Souza MFM, Gazal-Carvalho C, Malta DC, Alencar AP, Silva MMA, et al. Análise da mortalidade por homicídios no Brasil. Epidemiol Serv Saúde. 2007;16(1):7-18.

3- Kilsztajn S, Carmo MSN, Sugahara GTL, Lopes ES. Vítimas da cor: homicídios na região metropolitana de São Paulo, 2000. Cad Saúde Pública. 2005;21:1408-15.

4- Dias Júnior C S. O Impacto da Mortalidade por causas externas e dos homicídios na expectativa de vida: uma análise comparativa entre cinco regiões metropolitanas do Brasil. In: $2^{\circ}$ Congresso Português de Demografia, Lisboa, 2004.
5- Prefeitura Municipal de Londrina. Perfil do município de Londrina 2007 (ano base 2006). Londrina; 2007.

6- Peres MFT, Sants PC. Mortalidade por homicídios no Brasil na década de 90: o papel das armas de fogo. Rev Saúde Pública. 2005;39(1):58-66.

7- Hennington EA, Memeghel SN, Barros FS, Silva LB, Grano MS, Siqueira TP, et al. Mortalidade por homicídios em município da região sul do Brasil, 1996 a 2005. Rev Bras Epidemiol. 2008;11(3):43141.

8- Barata RB, Ribeiro MCSA, DE Sordi M. Desigualdades sociais e homicídios na cidade de São Paulo, 1998. Rev Bras Epidemiol. 2008;11(1):313.

9- Souza ER. Masculinização e violência no Brasil: contribuições para a reflexão no campo da saúde. Ciên Saúde Coletiva. 2005;10(1):59-70.

10- Macedo AC, Paim JC, Silva LMV, Costa MCN. 
Ferreira Filho OF, Cerci Neto A. Mortalidade por homicídios em Londrina, município do norte do Paraná.

Violência e desigualdade social: mortalidade por homicídios e condições de vida em Salvador, Brasil. Rev Saúde Pública. 2001;35: 15-22.

11- Tardiff K, Marzuk PM, Leon AC, Horsch CS, Stajicm, Portera $L$, et al. A profile of homicides on the streets and in the home of New York City. Public Health Rep. 1995;110(1):13-7.

12- Brasil. Casa Civil. Decreto n ${ }^{\circ} 6.488$, de 19 de junho de 2008 [citado em nov. 2008]. Disponível em: https://www.planalto.gov.br/ccivil_03/_ato20072010/2008/decreto/d6488.htm.

13- Souza ER, Assis SG, Silva C, Passos ME. Violência no município do Rio de Janeiro: áreas de risco e tendências de mortalidade entre adolescentes de 10 a 19 anos. Rev Panamer Saúde Pública. 1997;1:389-98.

Recebido em: 10/08/2010

Aprovado em: 06/09/2010 